\title{
Research on the Condition Maintenance Control of Electrical Equipment Based on Cloud Model and Improved TOPSIS Method
}

\author{
Li Zhiyu \\ Department of Electrical Engineering, North China Electric Power University, Baoding, China
}

Keywords: Electrical equipment, Condition maintenance control, Cloud model, Grey correlation degree, TOPSIS

\begin{abstract}
This paper presents a condition maintenance control method for electrical equipment combined with the advantages of cloud model and TOPSIS method improved by grey correlation degree. The cloud model is used to overcome the fuzziness and randomness of evaluation language set in the decision-making process. Besides, the TOPSIS method improved by grey correlation degree aims to solve the grey of evaluation language set. The examples analysis has verified the credibility of the proposed method.
\end{abstract}

\section{Introduction}

Equipment condition maintenance, based on the online technology providing equipment insulation and performance status, is to determine whether the equipment should be overhauled. Based on equipment status, condition maintenance is from the actual state of the device, taking the safety and economic basis into account[1]. From the development of the maintenance plan, an optimized program is selected, thereby the blind routine maintenance work is greatly reduced. Therefore, the study of the condition maintenance risk strategy can greatly avoid the waste of equipment maintenance resources and human and material resources, and is with broad application prospects and research significance[2].

In this paper, the cloud model is used to combine the fuzziness in the fuzzy theory with the randomness in the probability theory to realize the mutual mapping of qualitative and quantitative. Considering the ambiguity of the evaluation of experts and appraisers, and the incomplete information, the cloud model and the improved TOPSIS method are used to study the risk decision of condition maintenance control for electrical equipment.

\section{Cloud basic theory and grey correlation TOPSIS}

\section{(1) Cloud theory}

The digital features of the cloud are composed of expected value $E_{x}$, entropy $E_{n}$ and hyper entropy $H_{e}$. The expected value reflects the central value of domain which is the most representative point for qualitative concept. The entropy reflects the discretization degree of the cloud droplet and the accepted range in the domain. The hyper entropy represents the uncertainty measurement for entropy which is determined by the ambiguity and randomness of entropy. Thus, the cloud model is usually recorded as $C\left(E_{x}, E_{n}, H_{e}\right)$ [3].

In the decision-making evaluation, experts usually use natural language to express qualitative concepts[4]. Set natural language as $n$ scales, and $n$ is odd. According to the application requirements of this paper, the cloud computing process when $n$ is equal to 5 is introduced as follows:

Set the effective domain as $\left[X_{\min }, X_{\max }\right]$, the middle cloud as $C_{0}\left(E_{x 0}, E_{n 0}, H_{e 0}\right)$. Then the adjacent clouds are expressed as $C_{-2}\left(E_{x-2}, E_{n-2}, H_{e-2}\right), C_{-1}\left(E_{x-1}, E_{n-1}, H_{e-1}\right), C_{1}\left(E_{x 1}, E_{n 1}, H_{e 1}\right)$ and $C_{2}\left(E_{x 2}, E_{n 2}, H_{e 2}\right)$. The expected value of cloud model is calculated as follows: 


$$
\left\{\begin{array}{l}
E_{x-2}=X_{\text {min }} \\
E_{x-1}=(1-0.382) \frac{X_{\text {min }}+X_{\text {max }}}{2} \\
E_{x 0}=\frac{X_{\min }+X_{\text {max }}}{2} \\
E_{x 1}=(1+0.382) \frac{X_{\text {min }}+X_{\text {max }}}{2} \\
E_{x 2}=X_{\max }
\end{array}\right.
$$

The entropy value of cloud model is calculated as follows:

$$
\left\{\begin{array}{l}
E_{n-1}=E_{n 1}=\frac{0.382\left(X_{\text {max }}-X_{\text {min }}\right)}{6} \\
E_{n 0}=0.618 E_{n 1} \\
E_{n-2}=E_{n 2}=\frac{E_{n 1}}{0.618}
\end{array}\right.
$$

The hyper entropy value of cloud model is calculated as follows:

$$
\left\{\begin{array}{l}
H_{e-1}=H_{e 1}=\frac{H_{e 0}}{0.618} \\
H_{e-2}=H_{e 2}=\frac{H_{e 1}}{0.618}
\end{array}\right.
$$

According to the formula (1) to (3), the quantitative expression of the corresponding cloud model can be obtained. At this time, the concept of qualitative description can be transformed into quantitative index, which realizes the mapping of qualitative and quantitative. Because the experts who evaluate the decision are not the only one, the experts have differences according to their own subjective judgments[5]. Therefore, when the natural language is expressed, the cloud models are different, and the concept of floating cloud model is needed. When there are $k$ experts on the qualitative indicators of language evaluation, $k$ language value cloud models are generated. The aggregation cloud model is shown as follows:

$$
\begin{aligned}
& E_{x}=w_{1} E_{x 1}+w_{2} E_{x 2}+\cdots+w_{k} E_{x k} \\
& E_{n}=\frac{w_{1} E_{x 1} E_{n 1}+w_{2} E_{x 2} E_{n 2}+\cdots+w_{k} E_{x k} E_{n k}}{E_{x 1}+E_{x 2}+\cdots+E_{x k}} \\
& H_{e}=\sqrt{H_{e 1}^{2}+H_{e 2}^{2}+\cdots+H_{e k}^{2}}
\end{aligned}
$$

Where $w_{i}$ is the weight of evaluation value scored by each expert, and $w_{1}+w_{2}+\cdots+w_{k}=1$.

(2) Grey correlation TOPSIS

The grey correlation degree is a good approach to analyze the change of the curve and measure the similarity of the curve shape[6]. When TOPSIS method is applied to solves the multi-index decision problem, the uncertainty of quantitative relationship between factors is not taken into account. Thus, The TOPSIS method based on gray relational degree combines the distance scale and the gray relational degree of the traditional TOPSIS method, and constructs a new relative degree of proximity to realize the evaluation of the program[7-8].

Set $m$ programs, each program has $n$ indicators. The indicator value is $x_{i j}$, and the decision matrix is $X=\left(x_{i j}\right)_{m \times n}$. According to constructing the positive and negative ideal solutions $\left(U_{0}^{+}, U_{0}^{-}\right)$ of programs, the distance and grey relational degree between program $i$ and positive, negative ideal solutions can be obtained. After weighting the distance and grey correlation degree, the relative proximity of programs can be obtained. According to the size of relative proximity, the 
programs can be sorts. The lager the proposed value, the program is better.

\section{The risk decision of condition maintenance for transformers based on cloud model and grey relation TOPSIS}

(1) The conversion of indicators between qualitative and quantitative

In this paper, the effective domain of cloud model is set as $[0,1]$, the hyper entropy of intermediate cloud is 0.005 . The qualitative concepts are expressed as "good", "better", "middle", "poor" and "very poor". According to the golden section rate method in cloud theory, the corresponding cloud model can be obtained, as shown in Table 1.

Table 1 The qualitative concepts and theirs cloud models

\begin{tabular}{|c|c|}
\hline Qualitative concept & Cloud model \\
\hline Good & $(1,0.104,0.031)$ \\
\hline Better & $(0.691,0.064,0.008)$ \\
\hline Middle & $(0.5,0.039,0.005)$ \\
\hline Poor & $(0.309,0.064,0.008)$ \\
\hline Very poor & $(0,0.104,0.013)$ \\
\hline
\end{tabular}

(2) Decision matrix processing

According to the expert's natural language evaluation, $m$ programs can be obtained, and the decision-making matrix is $D=\left(d_{i j}\right)_{m \times n}$. Because the index values are divided into the benefit type index and the cost type index, and each index dimension is different. The vector normalization method is used to standardize the indexes, and the standardized decision matrix $Y=\left(y_{i j}\right)_{m \times n}$ can be obtained.

$$
Y_{i j}=\frac{d_{i j}}{\sqrt{\sum_{i=1}^{m} d_{i j}^{2}}}, \quad i=1,2, \cdots, m, \quad j=1,2, \cdots, n
$$

The entropy method is used to compute indicators' weights $W=\left(w_{1}, w_{2}, \cdots, w_{n}\right)$. And the weighted and standardized matrix is shown as follows:

$$
G=W Y=\left[\begin{array}{cccc}
g_{1}(1) & g_{1}(2) & \cdots & g_{1}(n) \\
g_{2}(1) & g_{2}(2) & \cdots & g_{2}(n) \\
\vdots & \vdots & \ddots & \vdots \\
g_{m}(1) & g_{m}(2) & \cdots & g_{m}(n)
\end{array}\right]
$$

(3) Determine the positive and negative ideal solution and distances

The positive ideal solution:

$$
\left.U_{0}^{+}=\left\{\left(\max _{1 \leq i \leq m} g_{i}(j), j \in J^{+}\right), \min _{1 \leq i \leq m} g_{i}(j), j \in J^{-}\right)\right\}=\left(u_{0}^{+}(j)\right)
$$

The negative ideal solution:

$$
U_{0}^{-}=\left\{\left(\min _{1 \leq i \leq m} g_{i}(j), j \in J^{+}\right),\left(\max _{1 \leq i \leq m} g_{i}(j), j \in J^{-}\right)\right\}=\left(u_{0}^{-}(j)\right)
$$

Where $J^{+}$represents the benefit type index and $J^{-}$represents the cost type index.

The distance from program $i$ to the positive ideal solution:

$$
D_{i}^{+}=\sqrt{\sum_{j=1}^{n}\left[g_{i}(j)-u_{0}^{+}(j)\right]^{2}}, \quad i=1,2, \cdots, m
$$

The distance from program $i$ to the negative ideal solution: 


$$
D_{i}^{-}=\sqrt{\sum_{j=1}^{n}\left[g_{i}(j)-u_{0}^{-}(j)\right]^{2}}, \quad i=1,2, \cdots, m
$$

(4) The relative proximity calculation of each programs

The distance and grey correlation degree are taken non-dimensional process, and the formula is as follows:

$$
B=\frac{B_{i}}{\max _{1 \leq i \leq m} B_{i}}
$$

Where $B_{i}$ represents $D_{i}^{+(-)}$and $R_{i}^{+(-)}$. Combing the dimensionless distance and the grey correlation degree, the comprehensive distances of programs can be obtained. $\alpha$ and $\beta$ are the preference degree for the position and shape of the decision maker, and $\alpha+\beta=1$.

$$
\left\{\begin{array}{l}
S_{i}^{+}=\alpha D_{i}^{-}+\beta R_{i}^{+} \\
S_{i}^{-}=\alpha D_{i}^{+}+\beta R_{i}^{-}
\end{array}\right.
$$

Calculate the relative proximity $C_{i}=\frac{S_{i}^{+}}{S_{i}^{+}+S_{i}^{-}}, \quad i=1,2, \cdots, m$. The lager the proposed value, the program is better.

\section{Example analysis}

In this paper, a transformer maintenance is selected as study object. There are three alternative maintenance programs, as shown in Table 2.

Table 2 Alternative maintenance programs

\begin{tabular}{|c|c|c|c|}
\hline No. & M1 & M2 & M3 \\
\hline Program & $\begin{array}{c}\text { Targeted } \\
\text { maintenance }\end{array}$ & $\begin{array}{c}\text { Advance } \\
\text { overhaul }\end{array}$ & $\begin{array}{c}\text { Do not arrange maintenance, continue to } \\
\text { track maintenance, shorten the tracking } \\
\text { cycle, to overhaul period and then } \\
\text { overhaul }\end{array}$ \\
\hline
\end{tabular}

The evaluation index system is shown in Table 3.

Table 3 Evaluation index system

\begin{tabular}{|c|c|}
\hline Type & Indicator \\
\hline \multirow{2}{*}{ Benefit type } & Maintenance technical level $\mathrm{c}_{1}$ \\
\cline { 2 - 2 } & Maintenance effect $\mathrm{c}_{2}$ \\
\hline \multirow{3}{*}{ Cost type } & Maintenance integrated costs $\mathrm{c}_{3}$ \\
\cline { 2 - 2 } & The impact of maintenance on production $\mathrm{c}_{4}$ \\
\cline { 2 - 2 } & Maintenance risk $\mathrm{c}_{5}$ \\
\cline { 2 - 2 } & The Effect of transformer fault on system security $\mathrm{c}_{6}$ \\
\hline
\end{tabular}

Three experts are invited to evaluate the three programs, and the expected values of the cloud model are obtained according to formula (4). The weight of each program is $1 / 3$, and the decision matrix is as follows:

$$
D=\left[\begin{array}{llllll}
0.9273 & 0.8295 & 0.3078 & 0.3078 & 0.3078 & 0.5000 \\
0.8295 & 0.8103 & 1.0000 & 0.9273 & 0.8103 & 0.8103 \\
0.8103 & 0.6464 & 0.5861 & 0.6464 & 0.5000 & 0.6922
\end{array}\right]
$$

The entropy method is used to calculated the weights of indicators.

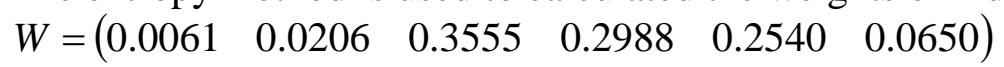


The above decision matrix is weighted and standardized:

$$
G=\left[\begin{array}{llllll}
0.0026 & 0.0097 & 0.0044 & 0.0670 & 0.0781 & 0.0235 \\
0.0023 & 0.0095 & 0.2472 & 0.2019 & 0.2055 & 0.0380 \\
0.0022 & 0.0076 & 0.0084 & 0.1407 & 0.1268 & 0.0325
\end{array}\right]
$$

The positive ideal solution is as follows:

$$
U_{0}^{+}=\left(\begin{array}{llllll}
0.0026 & 0.0097 & 0.0044 & 0.0670 & 0.0781 & 0.0235
\end{array}\right)
$$

The negative ideal solution is as follows:

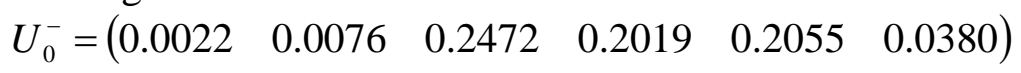

According to formula (11) to (18), the program distance value and grey correlation degree are obtained.

$$
\begin{aligned}
& D_{i}^{+}=\left(\begin{array}{lll}
0.0000 & 0.0936 & 0.0907
\end{array}\right) \\
& D_{i}^{-}=\left(\begin{array}{lll}
0.0936 & 0.0000 & 0.0207
\end{array}\right) \\
& R_{i}^{+}=\left(\begin{array}{lll}
0.8610 & 0.6213 & 0.9297
\end{array}\right) \\
& R_{i}^{-}=\left(\begin{array}{lll}
0.8816 & 0.8814 & 0.7960
\end{array}\right)
\end{aligned}
$$

According to formula(19), the above values are normalized. Combining the grey correlation degree and ideal solution distances of three programs, $\alpha=\beta=0.5$, the comprehensive distance of three program can be obtained. Then, calculate the relative proximity shown in Table 4.

Table 4 The comprehensive distance and relative proximity of three programs

\begin{tabular}{|c|c|c|c|}
\hline Program & M1 & M2 & M3 \\
\hline$S_{i}^{+}$ & 0.9631 & 0.3341 & 0.6106 \\
\hline$S_{i}^{-}$ & 0.5000 & 0.9998 & 0.9360 \\
\hline$C_{i}$ & 0.6582 & 0.2505 & 0.3948 \\
\hline
\end{tabular}

As can be seen from Table 4, the value order of relative proximity is M1> M3> M2. Therefore, the program M1 should be selected, namely according to the current detection of the fault, it is necessary to carry out maintenance of the transformer.

\section{Conclusion}

In the process of electrical equipment maintenance decision-making, the evaluation of uncertain language set of experts and technicians is gray and fuzzy. At the same time, the optimization of condition maintenance decision-making program involves many evaluation indexes, which is a multi-index uncertain language set decision process. Therefore, the maintenance decision based on the cloud model and the grey correlation TOPSIS method proposed in this paper has high reliability, which can provide the decision basis for the dispatching personnel to arrange the condition of the electric equipment and arrange the maintenance plan.

\section{References}

[1] Yang Liangjun, Xiong Xiaofu, Zhang Yuan. Research on condition-based maintenance policy of electric power equipment based on grey correlation degree and TOPSIS [J]. Power System Protection and Control, 2009(18): 74-78.

[2] Zhang Yuan, Xiong Xiaofu, Zhou Jiaqi, et al. Research on sequence of maintenance of circuit breakers based on grey fuzzy comprehensive evaluation [J]. Power System Technology, 2008, 32(8): 21-24.

[3] Li Ruqi, Tang Linquan, Ling Wuneng, et al. Risk decision-making based on cloud theory and 
prospect theory for conditional maintenance of power transformer [J]. Electric Power Automation Equipment, 2013( 2): 104-108.

[4]Ye Qiong, Li Shaowen, Zhang Youhua, et al. Cloud model and application overview [J]. Computer Engineering and Design, 2011, 32(12): 4198-4201.

[5] Fu Bin, Li Daoguo, Wang Mukuai. Review and prospect on research of cloud model [J]. Application Research and Computers, 2011, 28(2): 420-426.

[6] Sun Xiaodong, Jiao Yue, Hu Jinsong. Research on decision-making method based on gray correlation degree and TOPSIS [J]. Chinese Journal of Management Science, 2005, (4): 63-68.

[7] Qian Wuyong, Dang Yaoguo, Xiong Pingping, et al. Topsis Based on grey correlation method and its application[J]. System Engineering, 2008, 27(8): 124-126.

[8] Li Yanbin, Yu Xinyi, Wang Zhijie. Risk assessment on photovoltaic power generation project by grey correlation analysis and TOPSIS method[J]. Power System Technology, 2013, 37(6): 1514-1519. 